\title{
Beiträge zur systematischen Verwandtschaftslehre IX. Über Ammoniakverbindungen der Halogenide des zwej- wertigen Mangans und Eisens.
}

\author{
Von Wilhetim Brutz und Gustav F. Hüttig.
}

Mit 6 Figuren im Text.

Über die im Gleichgewichte mit gasförmigem Ammoniak auftretenden Verbindungen des zweiwertigen Kobalts und Nickels ist von W. Butz und seinen Mitarbeitern 1913 und 1914') berichtet worden. Die vorliegende Arbeit ist im engsten Anschlusse daran entstanden; sie bietet weder methodisch noch hinsichtlich der Verbindungstypen wesentlich Neues. Das war auch nicht zu erwarten. Wohl aber war es notwendig, das Zahlenmaterial aus jenen ersten Arbeiten durch die vollständige Untersuchung der einschlägigen Verbindungen der Mangan-, Eisen-, Kobalt-, Nickelgruppe zu ergänzen, wenn man es im Sinne der systematischen Verwandtschaftslehre $\left.{ }^{2}\right)$ auswerten wollte.

Ältere Arbeiten, die nicht systematisch auf die Aufsuchung von Gleichgewichten abzielten, vermochten auf unserem Gebiete nur Zufallstreffer zu bringen, wobei es an allerhand „Blindgängern“ nicht fehlte. Vou neueren Autoren hat Ephrarm in den Kreis seiner sehr zahlreichen physikalisch-chemischen Orientierungsversuche auch das Mangan und Eisen bezogen und über die Hexammine für unsere Zwecke nützliches Material beigebracht. Folgende Stoffe sind bisher beschrieben worden:

$\mathrm{Mn} \mathrm{Cl}_{2}$ nimmt nach Peress ${ }^{3}$ ) bei $-20^{\circ}$ 5.89 Mole $\mathrm{NH}_{3}$ auf und behält von diesen im Vakuum bei Zimmertemperatur 2.51 Mole. Das Präparat addiert wieder 3.79 Mole, regeneriert also das ursprüngliche Hexammin. Die Tension des Hexammins maß EpHrarm. ${ }^{4}$ ) Derselbe Autor ${ }^{5}$ ) bereitete aus Methylamin und entwässertem Manganchlorid ein Prüparat mit 5.6 Molen $\mathrm{NH}_{2} \cdot \mathrm{CH}_{3}$. Von $\mathrm{Mn} \mathrm{Br}_{2}$ ist durch Ephramm ${ }^{4}$ ) das Hexammin und eine Verbindung mit 5.7 Molen

1) W. Biltz, Z. phys. Chem. 82 (1913), 688; W. Biltz und B. Fetkenueuer, Z. anorg. Chem. 8:3 (1913), 163; 89 (1914), 97, 134.

2) W. Brutz, Z. anorg. Chem. 89 (1914), 141.

3) W. Petens, Ber. deutsch. chem. Ges. 42 (1909), 4833.

4) F. Epuraim, Z. phys. Chem. 81 (1913), 518.

5) F. Eprraim, Ber. deutsch. chem. Ges. 46 (1913), 3746.

Z. anorg. u. allg. Chein. Ba. 109. 
Methylamin ${ }^{1}$ ) untersucht worden. $\mathrm{Mn}_{2} \mathrm{~J}_{2}$ addiert nach Petzrss ${ }^{2}$ ) bei $-20^{\circ}$ 6.01 Mole $\mathrm{NH}_{3}$. Im Vakuum behielt es 5.13 Mole Ammoniak. Ein Pentammin ${ }^{3}$ ) existiert indessen, wie im nachfolgenden gezeigt wird, nicht. Die Tensionskurve des Hexammins ist von EpHrarm ${ }^{4}$ ) gemessen worden.

$\mathrm{FeCl}_{2} \cdot 6 \mathrm{NH}_{3}$ ist mehrfach bereits in der älteren Literatur beschrieben. ${ }^{5}$ ) Tensionsmessungen führten GIRARDET (6) und EpHraIM 4) aus; die beiderseitigen Beobachtungen stimmen, besonders in mittleren Druck-Temperaturgebieten, leidlich überein. Eine nicht näher charakterisierte Verbindung der Zusammensetzung $\mathrm{FeCl}_{2} \cdot 6.3 \mathrm{NH}_{3}$ erwähnt Girardet. Nach A. S. Mrclen ${ }^{7}$ ) verliert Hexammin im Vakuum $1 \mathrm{Mol} \mathrm{NH}_{3}$. Ein Pentammin existiert indessen, wie wir zeigen konnten, nicht; die Zusammensetzung ist ebenso wie bei dem schon erwähnten $\mathrm{MnJ}_{2} \cdot 5 \mathrm{NH}_{3}$ rein zufällig erreicht worden. Ebenso rein zufällig muB es erscheinen, wenn ein Bodenkörper von der Zusammensetzung $\mathrm{FeCl}_{2} \cdot 4 \mathrm{NH}_{3}{ }^{7}$ ) erhalten wurde. DaB aus Lösung eine Verbindung von Eisenchlorür mit 4 Molekülen Pyridin dargestellt werden konnte, $\left.{ }^{8}\right)$ kann hier nicht herangezogen werden, wo es sich lediglich um Gleichgewichtsprodukte innerhalb einer Ammoniakatmosphäre handelt. Auffällig erschien, daß sich aus Eisenchlorür mit gasförmigem Methylamin nur eine Gleichgewichtsverbindung mit 5 Molekülen bilden

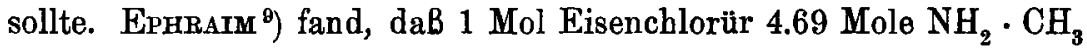
addieren und schlo $B$ daraus auf das Bestehen einer Verbindung $\mathrm{FeCl}_{2} \cdot 5 \mathrm{NH}_{2} \cdot \mathrm{CH}_{3}$, deren Tensionszahlen er angibt. Die nähere Untersuchung dieses Falles ergab, daB Ephraim sich lediglich durch die Langsamkeit der Addition hatte täuschen lassen und daB auch hier eine Verbindung mit 6 Molekülen gebildet wird. Somit machen

1) F. Ephraix, Ber. deutsch. chem. Ges. 46 (I913), 3746.

2) W. Peters, Ber. deutsch. chem. Ges. 42 (1909), 4833.

3) Die Verbindung $\mathrm{Mn}\left(\mathrm{NH}_{3}\right)_{5} \mathrm{~J}_{3}$ ist, freilich mit einem Fragezeichen versehen, in die Sammelliteratur, Lexikon der anorg. Verbindungen II, 116 übergegangen.

4) F. Ephratm, Z. phys. Chem. 81 (1913), 518.

5) Im Lexikon der anorg. Verb. II, 207 wird als fraglich auch eine Verbindung $\mathrm{FeCl}_{2} \cdot 7 \mathrm{NH}_{8}$ zitiert, ohne daB indessen an der angeführten Literaturstelle etwas darüber zu finden ist.

0) Girardet, Bull. Soc. chim. (4) 7 (1910),'1028; Chem. Zentralbl. 1911, I, 291.

7) A.S. Mrusr, Amer. chem.Journ.17 (1894), 570; Chem. Zentrbl. 1895, II, 960.

8) N. Costachesco und G. Spacu, Ann. scient. Univ. Jassy 7, 132; Chem. Zerstrabl. 1912, I, 1972.

9) F. Ephra1m, Ber. deutsch. chem. Ges. 46 (1913), 3749. 
die Eisenhalogenverbindungen hier nicht, wie EPHRAIM vermutete, eine Ausnahme. Es war dies übrigens umso unwahrscheinlicher, als die Tensionsmessungen der „Pentaverbindungen" sich denen der verwandten Hexaverbindungen durchaus anschlieBen, wie dies der Autor ausdrücklich anmerkt. $\mathrm{FeCl}_{2} \cdot 2 \mathrm{NH}_{3}$ ist von MILLER ${ }^{1}$ ) aus dem Hexammin im Wasserstoffstrom bei $100^{\circ}$ erhalten worden. Tensionsmessungen brachte GTRARDET ${ }^{2}$ ) bei, der das ganze Gebiet zwischen Hexammin und Eisenchlorür absuchte. Wir können die Existenz dieser Verbindungen bestätigen, müssen aber die Angaben GiRarders dahin ergänzen, dab auch ein Monammin besteht, von dem bisher nichts bekannt war.

Von Verbindungen aus Ammoniak und Eisenbromür war bisher nur ein Hexammin von EpHraIm ${ }^{3}$ ) erhalten und gemessen worden. Eisenjodür - Hexammin war von $J_{A C K S O N}$ und DeBBY ${ }^{4}$ ) dargestellt worden; seine Tension maB Ephraim. ${ }^{3}$ )

Unsere Versuchsmethoden waren die gleichen wie früher.") Es wurde zunächst durch Isothermen festgelegt, welche Verbindungen überhaupt im Gleichgewicht mit gasförmigem Ammoniak zwischen dem reinen Salz und dem bei Zimmertemperatur und einer Atmosphäre stabilen, höchsten Ammoniakat bestehen. Ob bei extremen Drucken und niedrigen Temperaturen noch höhere Ammoniakanlagerungsverbiudungen möglich sind, wäre Gegenstand einer besonderen Untersuchung. Für $\mathrm{MnJ}_{2}$ muB es als sicher gelten, daB sich zwischen $\mathrm{MnJ}_{2} \cdot 6 \mathrm{NH}_{3}$ und fiüssigem Ammoniak von $-80^{\circ}$ kein höheres festes Ammoniakat einschiebt. Die Apparatur war die gleiche, wie früher. Die Anwendung von Siedeflüssigkeiten zum Konstanthalten der Temperaturen bewährte sich bestens. Als Heizquelle benutzten wir kleine mit Chromindraht bewickelte elektrische Öfen, teils der Bequemlichkeit und Sicherheit halber, teils, weil es kein Gas gab. In den meisten Fällen wurden zur Festlegung der Isothermen mehrere Versuchsreihen angesetzt; in den Tabellen kennzeichnen sich die jeweils zu einer Reihe gehörenden Werte an der Gleichheit der Einwagen an ammoniakfreiem Salz. Die weiteren,

1) A.S. MILLER, Amer.Chem.Journ.17(1894), 570; Chem.Zentralbl.1895, II, 960.

2) Girardet, Bull. Soe. chim. (4) 7 (1910), 1028; Chem. Zentralbl. 1911 I, 291.

3) EpHram, Zeitschr. phys. Chem. 81 (1913), 520.

4) C. JACrsox und J. H. DrRBX, Amer. Chem. Journ. 24 (1899) 15; Chem. Zentralbl. 1900, II, 559.

5) W. Biltz und B. Fetrenhedrr, Zeitschr. anorg. Chem. 83 (1913), 164; 89 (1914) 98. B. Fetkenhedre, Disser. Univ. Berlin 1914. 
zur Charakterisierung der Tensionskurven aufgenommenen Druckwerte jeder Verbindung wurden in derselben Apparatur unter Wechsel der Heizflüssigkeiten bestimmt. Die Daver der Konstanz jeder Ablesung richtete sich nach der Dauer der Einstellung. Bei niedrigen Temperaturen und dann, wenn der Bodenkörper aus Mischkristallen bestand, war die Einstellung sehr träge und es wurde 12 und mehr Stunden gewartet, ehe man sich mit der Konstanz eines Wertes zufrieden gab. Bei glatter Einstellung genügte natürlich kürzere Konstanz. In der weitaus gröBten Zahl der Fälle lieb man die Einstellung sich ron unten, also bei wachsendem Druck vollziehen, weil die Einstellung von oben noch viel langsamer verläuft. Kontrollen der Umkehrbarkeit der Vorgänge wurden indes hinreichend eingeschaltet. Die Gehaltsbestimmung erfolgte, wie früher, durch Wägung des Bodenkörpers. Dabei fiel auf, daß regelmäBig nach Verwendung relativ hoher Temperatur ( $\alpha$ Bromnaphtalin 2789) das Leergewicht des Glaskörpers um einige Milligramme abnahm, vermutlich infolge Überhitzung des Schmiermittels im Schliffstück. Am Schlusse der Versuchsreihe wurde diese Korrektur festgestellt und nötigenfalls die Zusammensetzung des Bodenkörpers analytisch kontrolliert.

Besondere Sorgfalt muBte auf die Zusammensetzung des Ausgangsmaterials gelegt werden. Unsere Vorgänger begnügten sich zur Herstellung der wasserfreien Salze meist mit der Entwässerung der Hydrate. Das ist, falls sie nicht unter ganz besonderen Vorsichtsmakregeln bei sehr niedrigen Temperaturen im Hochvakuum sehr langsam eingeleitet wird, keine brauchbare Methode und selbst die größte Sorgfalt versagt bei besonders leicht hydrolysierbaren Stoffen. Zur Orientierung über den Hexammindruck mag es genügen, mit einem aus Oxyd, Hydroxyd, basischen Salzen, Ammoniumbalogenid, Hexammin, niederen Amminen und Mischkristallen bestehenden Gemenge zu arbeiten; aber für eine leidlich zuverlässige Aufnahme des ganzen Zustandsdiagrammes ist es völlig unzureichend. Das näbere über die Ausgangsstoffe wird bei den einzelnen Salzen mitgeteilt werden. Die Darstellung der Hexammine aus den reinen Salzen erfolgte stets im Apparat selbst. Die Salze wurden dazu vor der Wägung und Ammoniakabsorption nochmals bei geeigneten Temperaturen zur Entfernung von Wasserresten nachgetrocknet, die beim Nontieren des Apparats eingedrungen sein konnten. Da Ammoniak bei Zimmertemperatur vielfach nur sehr langsam absorbiert wird, kühlte man die Probe mit fester Kohlensäure und Alkohol oder einer anderen 
Kältemischung $a b$, so daß also die erste Sättigung unter Umständen zwischen flüssigem Ammoniak und Salz erfolgte. Man lieB dann bei Zimmertemperatur das etwa überschüssige Ammoniak abdunsten, und sorgte so dafür, daB das Ausgangsmaterial schlieBlich ein Gleichgewichtsprodukt aus gasförmigem Ammoniak und Salz darstellte. Die Salze nahmen durch die Ammoniakaufnahme bis Hexammin auserordentlich stark an Volumen zu, wie das alle früheren Beobachter ebenfalls angeben. Beim Abbau schwindet das Ammoniakat stark.

\section{Hanganchlorür-Ammoniakate.}

Manganchlorür von KaHLbuca wurde im Vakuum ${ }^{1}$ ) bei Zimmertemperatur, später bei $35^{0}$ vorgetrocknet, im Chlorwasserstoffstrome bei $120-130^{\circ}$ entwässert und endgültig im Vakuum über Àtznatron bei $140-150^{\circ}$ für den Versuch vorbereitet. Das blabrosa Pulver löste sich in Wasser mit neutraler Reaktion. Zwei verschiedene Präparate wurden analysiert:

1. $0.5727 \mathrm{~g}$ gaben $1.3166 \mathrm{~g} \mathrm{AgCl} ; 56.6 \% \mathrm{Cl}$

2. $0.2623 \mathrm{~g}$ gaben $0.5959 \mathrm{~g} \mathrm{AgCl} ; 56.1 \% \mathrm{Cl}$ ber. $56.3 \% \mathrm{Cl}$

Hexammin manganochlorid. Bei Zimmertemperatur sättigte sich Manganchlorür selbst innerhalb dreier Tage noch nicht völlig mit Ammoniak, weswegen zur Beschleunigung das oben geschilderte Verfahren mit fester Kohlensäure und Alkohol verwendet wurde, das in einigen Stunden zum Ziel führte.

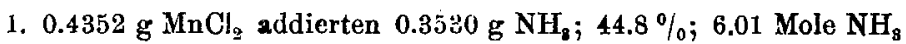

2. $0.4049 \mathrm{~g} \mathrm{MnCl}$, addierten $0.3265 \mathrm{~g} \mathrm{NH}_{3} ; 44.6 \% ; 5.96 \mathrm{Mole}^{\mathrm{N}} \mathrm{NH}_{3}$

3. $0.2813 \mathrm{~g} \mathrm{MnCl}_{2}$ addierten $0.2264 \mathrm{~g} \mathrm{NH}_{3} ; 44.6 \% ; 5.96 \mathrm{Mole} \mathrm{NH}_{3}$ ber. für $\mathrm{MnCl}_{2} \cdot 6 \mathrm{NH}_{3}: 44.8 \%$

Hexammin manganochlorid ist rein weiß. Ebenso die anderen Ammine. Eine Kontrolle des Abbaues durch makroskopische oder mikroskopische Betrachtung der Bodenkörper, wie sie sich in der Nickel-Kobaltreihe so nützlich erwiesen hatte, fiel somit hier und auch in den meisten folgenden Fällen weg.

a) Isotherme $\mathrm{zwischen} \mathrm{MnCl}_{2} \cdot 6 \mathrm{NH}_{3}$ und $\mathrm{MnCl}_{2} \cdot 2 \mathrm{NH}_{3}$. Der Abbau wurde mit Äthylalkohol als Heizflüssigkeit vorgenommen, wobei das hygroskopische Siedemittel entsprechend zu schützen war; alle Drucke wurden auf $76.5^{\circ}$, dem Siedepunkte bei $709 \mathrm{~mm}$ bezogen. ${ }^{2}$ )

1) Bei allen Trocknungen bewährte sich der von Haxs Mexen angegebene, von der Firma FraNz MüLler, Bonn, gelieferte Exsikkator bestens.

2) Vgl. Zeitschr. anorg. Chem. 89 (1914), 99. 
Tabelle 1.

$\mathrm{MnCl}_{2} \cdot 6 \mathrm{NH}_{3} \rightleftharpoons \mathrm{MnCl}_{2} \cdot 2 \mathrm{NH}_{8}+4 \mathrm{NH}_{8}$

\begin{tabular}{c|c|c|c|c}
\hline \hline $\mathrm{Nr}$. & $\mathrm{g} \mathrm{MnCl}_{2}$ & $\mathrm{~g} \mathrm{NH}_{3}$ & Mole $\mathrm{NH}_{3}$ & p mm korr. $\left.{ }^{1}\right)$ \\
\hline 1 & & & & \\
\hline 2 & 0.4049 & 0.3057 & 5.59 & 491 \\
3 & 0.4049 & 0.2872 & 5.25 & 471 \\
4 & 0.4049 & 0.2625 & 4.80 & 471 \\
5 & 0.2813 & 0.0757 & 1.99 & 474 \\
6 & 0.2813 & 0.0718 & 1.89 & 325 \\
7 & 0.2813 & 0.0692 & 1.82 & 50.8 \\
& 0.2813 & 0.0681 & 1.79 & 7.3
\end{tabular}

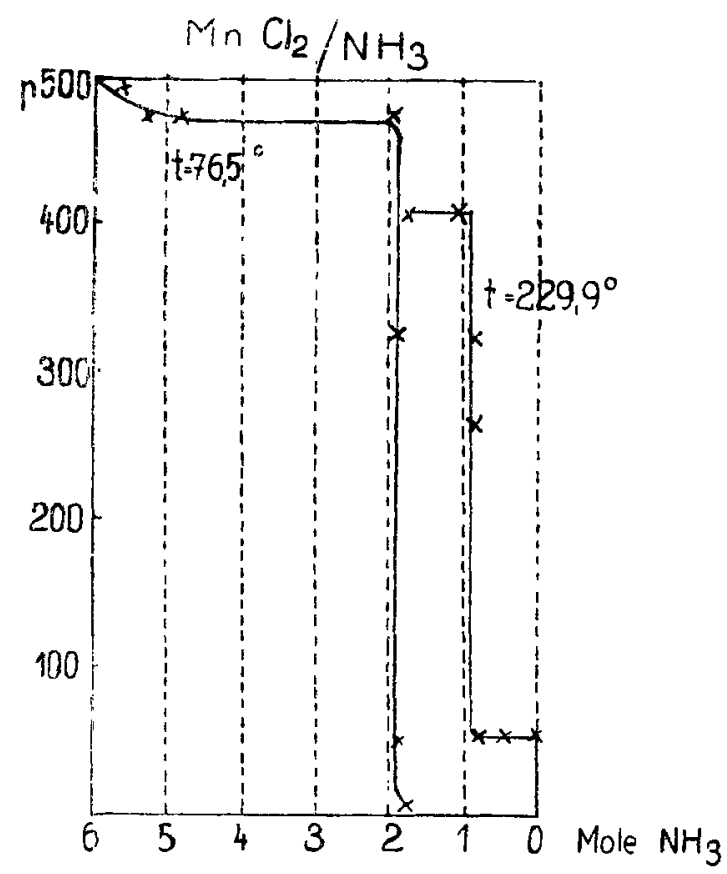

Fig. 1.

Sämtliche Versuche wurden im Abbau von unten eingestellt. Nur Nr. 5 im Aufbau d. h. es wurde einer bereits unter 1.89 Mole abgebauten Probe $\mathrm{NH}_{3}$ aufs neue addiert und dann dieses Produkt der Einstellung im Tensimeter unterworfen, doch so, daB auch hier die Kinstellung von unten erfolgte. Die Einstellung ging sehr träge von statten, teils wegen der relativ niedrigen Temperatur, teils wegen der Bildung der Mischkristalle. Die Druckkonstanz betrug bei den entscheidenden Versuchen mindestens 12 Stunden.

1) Vgl. Zeitschr, anorg. Chem. 89 (1914), 99; $\mathrm{p}$ in $\mathrm{mm} \mathrm{Hg.}$ 
Die Isotherme sinkt, wie die Tabelle und Fig. 1 zeigen, von der Zusammensetzung des Hexammins zunächst merklich und bleibt dann bis zum Diammin horizontal. Die Existenz des Diamminmanganochlorids ist damit erwiesen. Die Kurve bietet ein ähnliches Bild wie beim $\mathrm{CoCl}_{2} \cdot 6 \mathrm{NH}_{3}$ und $\mathrm{CoBr}_{2} \cdot 6 \mathrm{NH}_{2}$, d. h. der Abbau des Hexammins führt zu Mischkristallen von Hexammin und Diammin. Eine Verbindung mittlerer Zusammensetzung besteht nicht.

b) Isotherme zwischen $\mathrm{MnCl}_{2} \cdot 2 \mathrm{NH}_{3}, \mathrm{MnCl}_{2} \cdot \mathrm{NH}_{3}$ und $\mathrm{MnCl}_{2}$. Heizflüssigkeit Thymol vom Siedepunkte 229.9 $(710 \mathrm{~mm})$.

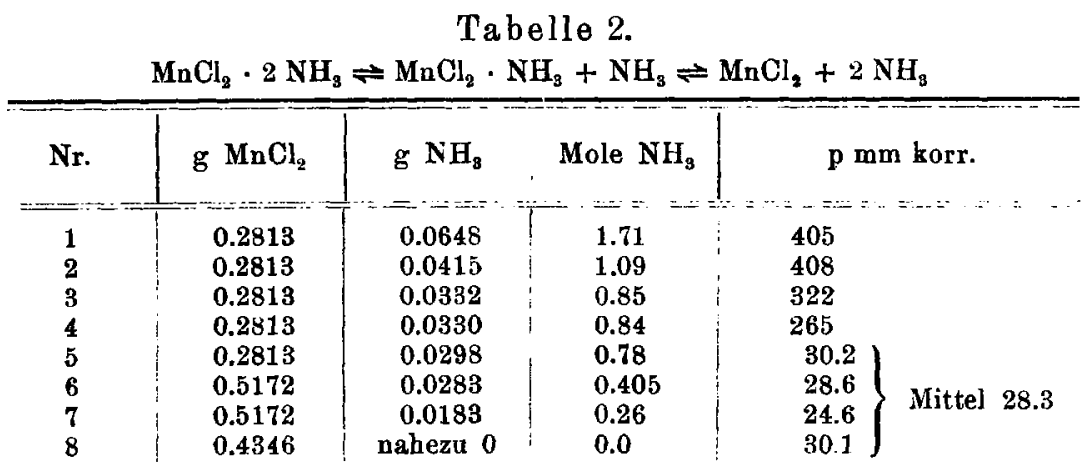

Einstellung von unten. Bei Versuch 6 von unten und oben, dieser Druck zeigte $18 \mathrm{St}$. Konstanz, dürfte besonders zuverlässig sein und deckt sich auch mit dem Mittelwert. Die Einstellung erfolgte durchweg schneller als bei den Versuchen der Tabelle 1 wegen des Fehlens von Mischkristallen und wegen der höheren Temperatur Die Isotherme erweist die Existenz des Monammin manganochlorids. Andere Ammine, als die genannten, bestehen innerhalb der beobachteten Temperatur- und Druckgebiete im Gleichgewicht mit Ammoniakgas nicht.

c) Tensionswerte von $\mathrm{MnCl}_{2} \cdot 6 \mathrm{NH}_{3}$. Neben den $t, p$-Werten sind unter $Q$ die Dissoziationswärmen nach der Näherungsgleichung von Nernst ausgerechnet. Über die Bedeutung dieser Werte und ihren Ersatz durch genauere findet sich in der nachfolgenden Abhandlung Näheres.

Tabelle 3.

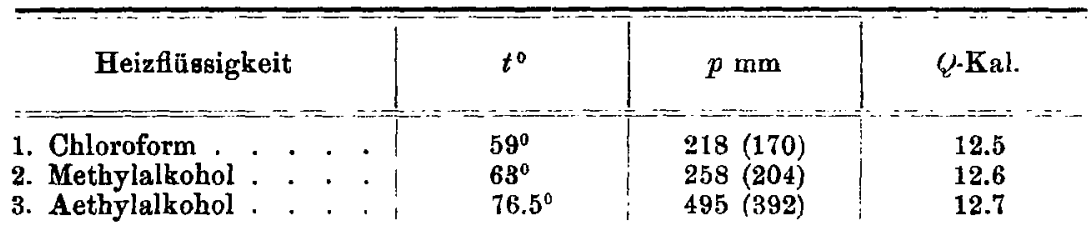


Um die erniedrigende Wirkung der Mischkristallbildung auf die Drucke möglichst auszuschalten, sind die Werte 1 und 2 in Konzentrationen möglichst dicht am Hexammin ermittelt; 3 ist der aus der Isotherme extrapolierte Wert. Neben unseren $p$-Werten sind geklammert die Werte von EPHRAIM angegeben; sie liegen wesentlich tiefer.

d) Tensionswerte von $\mathrm{MnCl}_{2} \cdot 2 \mathrm{NH}_{3}$.

Tabelle 4.

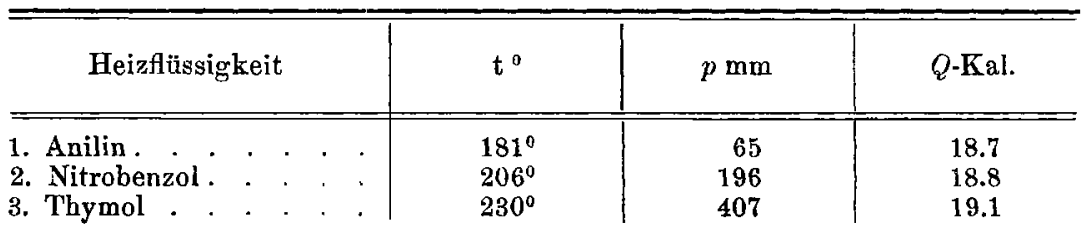

Wert 1 stellt das Mittel von drei Werten dar, die in einer Konzentration zwischen 1.4 and $1.8 \mathrm{Mol} \mathrm{NH}_{3}$ aufgenommen wurden, Wert 3 das Mictel der beiden Isothermenwerte.

e) Tensionswerte ron $\mathrm{MnCl}_{2} \cdot \mathrm{NH}_{3}$.

Tabelle 5.

\begin{tabular}{l|c|c|c}
\hline Heizflissigkeit & $t^{0}$ & $p \mathrm{~mm}$ & $Q$-Kal. \\
\hline \hline 1. Naphthalin . . . . . & $\mathbf{2 1 5}^{\circ}$ & $\mathbf{9 . 4}$ & $\mathbf{2 2 . 1}$ \\
2. Thymol . . . . & $230^{\circ}$ & 28 & 21.75 \\
3. a-Bromnaphthalin. & $278^{\circ}$ & 95 & 22.7
\end{tabular}

Wert 2 ist das Mittel aus vier guten Werten der Isothermen und zweifellos der zuverlässigste dieser Reihe.

\section{Manganbromür-Ammoniakate.}

Wasserhaltiges Manganbromür von KaHLBAUM wurde im Vakuum bei Zimmertemperatur vorgetrocknet und des weiteren im Vakuum 17 Stunden bei weniger als $60^{\circ}$, zweieinhalb Tage bei $70^{\circ}$, 2 Stunden bei $95^{\circ}$ und schlieblich 2 Stunden bei $150^{\circ}$ getrocknet. Das Präparat sah rosa aus, war jedoch tiefer gefärbt als das wasserfreie Chlorür.

$$
\begin{array}{r}
0.2954 \mathrm{~g} \text { gaben } 0.5162 \mathrm{~g} \mathrm{AgBr} ; 74.4 \% \mathrm{Br} \\
\text { ber. } 74.4 \% \mathrm{Br}
\end{array}
$$

Hexammin manganobromid. Nach demselben Verfahren, wie beim Chlorür, sättigte sich das wasserfreie Salz in 6 Stunden mit Ammoniak. 
1. $0.3528 \mathrm{~g} \mathrm{MnBr}_{2}$ addierten $0.1679 \mathrm{~g} \mathrm{NH}_{3} ; 32.2 \%$; $6.01 \mathrm{Mole} \mathrm{NH}_{3}$

2. $0.3937 \mathrm{~g} \mathrm{MnBr}_{2}$ addierten $0.1859 \mathrm{~g} \mathrm{NH}_{3} ; 32.1 \% ; 5.97 \mathrm{Mole}_{\mathrm{NH}}$

3. $0.6552 \mathrm{~g} \mathrm{MnBr}_{2}$ addierten $0.3095 \mathrm{~g} \mathrm{NH}_{3} ; 32.1 \%$; $5.98 \mathrm{Mole}_{\mathrm{NH}}$ ber. für: $\mathrm{MnBr}_{2} \cdot 6 \mathrm{NH}_{3}: 32.2 \%$

a) Isotherme zwischen $\mathrm{MnBr}_{2} \cdot 6 \mathrm{NH}_{3}$ und $\mathrm{MnBr}_{2} \cdot 2 \mathrm{NH}_{3}$. Heizflüssigkeit Toluol vom Siedepunkte $107.3^{\circ}$ (707 mm).

Tabelle 6.

$\mathrm{MnBr}_{2} \cdot 6 \mathrm{NH}_{3} \rightleftharpoons \mathrm{MnBr}_{2} \cdot 2 \mathrm{NH}_{3}+4 \mathrm{NH}_{3}$.

\begin{tabular}{c|c|c|c|c}
\hline $\mathrm{Nr}$ & $\mathrm{g} \mathrm{MnBr}_{2}$ & $\mathrm{~g} \mathrm{NH_{3 }}$ & Mole $\mathrm{NH}_{3}$ & $\mathrm{p} \mathrm{mm} \mathrm{korr.}$ \\
\hline 1 & 0.6552 & 0.3009 & 5.80 & \\
\hline 2 & 0.3937 & 0.1802 & 5.78 & $\mathbf{3 0 5}$ \\
3 & 0.3937 & 0.1710 & 5.49 & 290 \\
4 & 0.6552 & 0.2638 & 5.09 & 299 \\
5 & 0.7787 & 0.2702 & 4.38 & 293 \\
6 & 0.6552 & 0.1289 & 2.49 & 284 \\
7 & 0.7787 & 0.1267 & 2.06 & 267 \\
8 & 0.6552 & 0.0990 & 1.91 & 228 \\
9 & 0.6552 & 0.0990 & 1.91 & 3,1
\end{tabular}

Die Einstellung erfolgte stets von unten; die Konstanz ging bis zu 20 Stunden.

b) Is otherme zwischen $\mathrm{MnBr}_{2} \cdot 2 \mathrm{NH}_{3}, \mathrm{MnBr}_{2} \cdot \mathrm{NH}_{3}$ und $\mathrm{MnBr}_{2}$. Heizflüssigkeit Thymol vom Siedepunkte $230.3^{\circ}(715 \mathrm{~mm})$.

Tabelle 7.

$\mathrm{MnBr}_{2} \cdot 2 \mathrm{NH}_{3} \rightleftharpoons \mathrm{MnBr}_{3} \cdot \mathrm{NH}_{3}+\mathrm{NH}_{3} \rightleftharpoons \mathrm{MnBr}_{3}+2 \mathrm{NH}_{3}$.

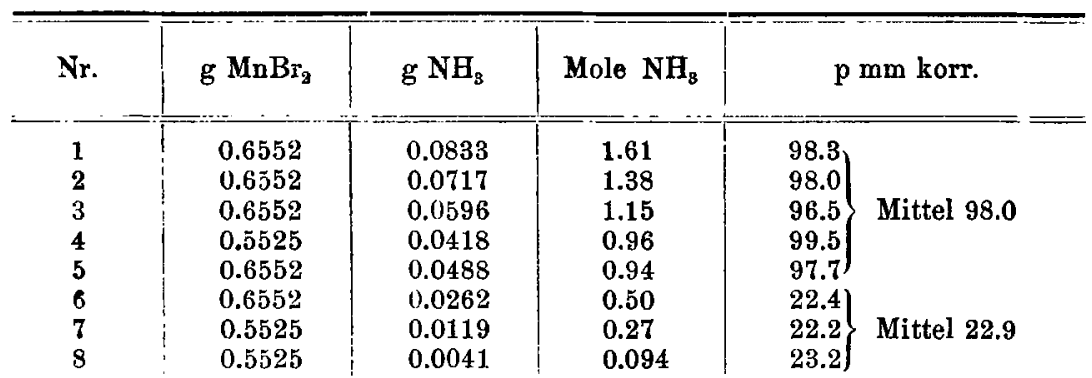

Die Konstanz betrug 3-14 Stunden. Außer bei Versuch 7 und 8 wurden sämtliche Werte von unten her erreicht. Die Fig. 2 beweist uns die Existenz von Diammin manganobromid und Monammin manganobromid und zeigt zugleich an, dab diese neben Hexammin die einzigen Ammoniakate unseres Zustandsdia- 
grammes sind. Mischkristallbildung zwischen Hexammin und Diammin ist auch hier wieder merkbar. Alle drei Ammoniakate sind rein weiB.

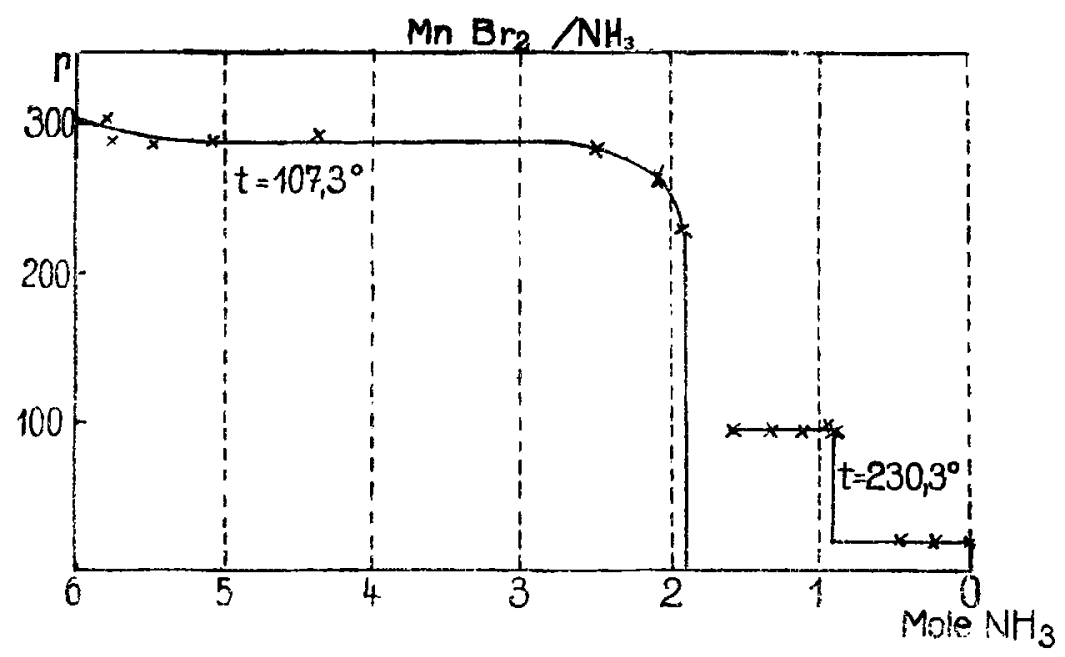

Fig. 2.

c) Tensionswerte von $\mathrm{MnBr}_{2} \cdot 6 \mathrm{NH}_{3}$.

Tabelle 8.

\begin{tabular}{|c|c|c|c|c|}
\hline Heizflüssigkeit & $t^{0}$ & $\mathbf{p} \mathbf{m m}$ & $Q$-Kal. & Bemerkung \\
\hline Toluol ... & $\begin{array}{l}107^{\circ} \\
107^{\circ}\end{array}$ & $\begin{array}{l}305 \\
275\end{array}$ & $\begin{array}{l}14.26 \\
14.34\end{array}$ & $\begin{array}{l}\text { Aus d. Isothermen extrapoliert } \\
\text { Nach EPHRIM interpoliert }\end{array}$ \\
\hline
\end{tabular}

Den weiteren Berechnungen wird der von uns beobachtete Wert zu Grunde gelegt.

d) Tensionswerte von $\mathrm{MnBr}_{2} \cdot 2 \mathrm{NH}_{3}$.

Tabelle 9 .

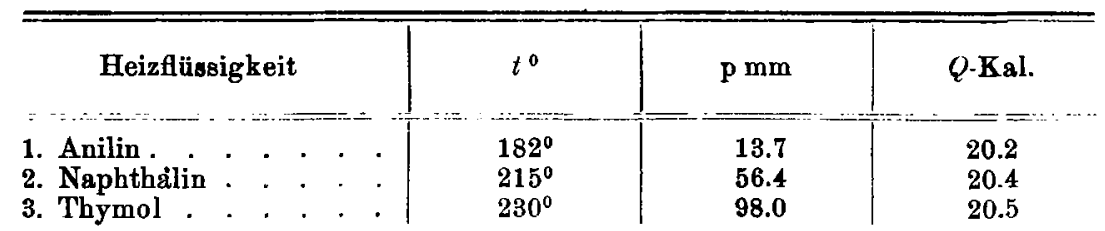

Der Wert 1 wurde in einer Konzentration von 1.2 bis $1.3 \mathrm{Mol} \mathrm{NH}_{3}$ aufgenommen; er blieb 12 Stunden konstant. Wert 3 ist das Mittel aus den Isothermenwerten. 
e) Tensionswerte von $\mathrm{MnBr}_{2} \cdot \mathrm{NH}_{3}$.

Tabelle 10.

\begin{tabular}{|c|c|c|c|}
\hline Heizflüssigkeit & $t^{0}$ & $\mathbf{p} \mathbf{m m}$ & Q-Kal. \\
\hline 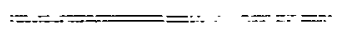 & & $=\because$ & $\cdots \cdots, \quad \cdots=$ \\
\hline $\begin{array}{l}\text { 1. Naphthalin . } . \\
\text { 2. Thymol . } \\
\text { 3. } \alpha \text {-Bromnaphthalin. }\end{array}$ & $\begin{array}{l}215^{0} \\
230^{\circ} \\
278^{\circ}\end{array}$ & $\begin{array}{l}12.3 \\
22.9 \\
114\end{array}$ & $\begin{array}{l}21.8 \\
21.95 \\
22.5\end{array}$ \\
\hline
\end{tabular}

Die Werte 1 und 3 blieben 12 bezw. 20 Stunden konstant; die Konzentration lag bei 0.5 bis $0.7 \mathrm{Mol} \mathrm{NH}_{3}$. Wert 2 ist das Mittel aus den Isothermenwerten.

\section{Manganjodür-Ammoniakate.}

Reines Manganjodür scheint noch nicht dargestellt worden zu sein. Peters ${ }^{1}$ ) und EpHranc ${ }^{2}$ ) trockneten wasserhaltige Präparate; doch überzeugten wir uns, daB bei noch so sorgfältigem Vorgehen, wie das bei den vorhergehenden Salzen beschrieben ist, stets um etwa 5\% Jod zu arme Präparate entstehen. F. DuceLLIEz ${ }^{3}$ ) behandelte Manganpulver in Gegenwart von wasserfreiem Äther mit Jod; sein Präparat wird als weißes Pulver beschrieben, während ein wasserfreies Manganjodür, wie wir es erhielten, rosa gefärbt ist. Es liegt nahe, anzunehmen, daB das Präparat von Docencrez ätherhaltig war, wenngleich die von DUCELLIEz ansgeführte Analyse nichts davon merken läßt. Aber die entsprechend hergestellten Bromide des Kobalts und Nickels sind nach Duceuliez ätherhaltig ${ }^{4}$ ) und ein besonderer Versuch zeigte uns, daB in der Tat unser wasserfreies; rosa gefärbtes Manganjodür mit Äther überschichtet sogleich weil wird.

Unschwer läBt sich Hexammin manganojodid auf nassem Wege erhalten und dieser Stoff scheint uns auch das bequemste Ausgangsmaterial für wasserfreies Manganjodür zu sein. Zur Darstellung dieses Hexammins löst man das wasserhaltige Manganjodüı bei Wasserbadtemperatur in starker, portionsweise zugegebener Ammoniakflüssigkeit (auf $15 \mathrm{~g}$ Salz etwa $500 \mathrm{ccm}$ Flüssigkeit). Die Lösung ist farblos, aber durch braune und helle Teilchen getrübt; sie wird durch einen Heißwassertrichter in eisgekühlten, durch Aus-

1) W. Pennrs, Ber. deutsch. chem. Ges. 42, (1909), 4833. Das Präparat wird als rotbraun beschrieben.

2) F. Ephratm, Ber. deutsch. chem. Ges. 45, (1912), 1330.

5) F. Dockuliez, Bull. Soc.chim. (4), 13 (1913),815; Chem. Zentrlbl. 1913 II, 1554.

4) F. Dodellrez u. A.RayNaUd, C.R.15\$(1914)2002; Chem.Zentrlbl.1914, II,609. 
kochen von Luft befreiten und mit Ammoniak gesättigten Alkohol einfiltriert und dadurch gefällt. Bei Luftabschluß läBt man die Kristallisation unter Eiskühlung zu Ende gehen und sammelt das Präparat durch Absaugen unter möglichster Vermeidung von Luftzutritt; es wird ohne Erwärmung im Vakuumexsikkator über Ätznatron und etwas Salmiak getrocknet und bildet ein rein weiBes, aus mikroskopischen, wohl ausgebildeten Oktaedern bestehendes Kristallpulver. $A n_{i}$ der Luft wird es in einigen Minuten bräunlich, zerflieBt aber in einigen Stunden nicht. Zur Analyse wurde das Ammoniak abdestilliert und titriert.

1. $0.6110 \mathrm{~g}$ verbrauchten $89.8 \mathrm{cem} 1 / 10 \mathrm{n} \mathrm{H}_{2} \mathrm{SO}_{4} ; 24.8 \% \mathrm{NH}_{3}$

2. $0.3278 \mathrm{~g}$ verbrauchten $48.0 \mathrm{ccm} 1_{1 / 10} \mathrm{n} \mathrm{H}_{2} \mathrm{SO}_{4} ; 24.9 \% \mathrm{NH}_{3}$

3. $0.6421 \mathrm{~g}$ verbrauchten $93.7 \mathrm{ccm} 1 / 10 \mathrm{n} \mathrm{H}_{2} \mathrm{SO}_{4} ; 24.8 \% \mathrm{NH}_{3}$ ber. für $\mathrm{MnJ}_{2} \cdot 6 \mathrm{NH}_{3}: 24.87 \% \mathrm{NH}_{3}$

a) Isotherme zwischen $\mathrm{MnJ}_{2} \cdot 6 \mathrm{NH}_{3}$ und $\mathrm{MnJ}_{2} \cdot 2 \mathrm{NH}_{3}$. Bei der Empfindlichkeit des Hexammins gegenüber Feuchtigkeit bei höherer Temperatur wurden die Gehaltsermittlungen des Bodenkörpers durch eine Analyse des Endproduktes (Tabelle 12, Nr. 4) kontrolliert und danach eine kleine Korrektur angebracht.

Heizflüssigkeit Brombenzol vom Siedepunkt $153.6^{\circ}$ (713 mm).

Tabelle 11.

$$
\mathrm{MnJ}_{2} \cdot 6 \mathrm{NH}_{3} \rightleftharpoons \mathrm{MnJ}_{2} \cdot 2 \mathrm{NH}_{3}+4 \mathrm{NH}_{3}
$$

\begin{tabular}{c|c|c}
\hline Nr. & Mole $\mathrm{NH}_{3}$ & $p$ mm korr. \\
\hline 1 & 5.58 & 312 \\
2 & 4.73 & 316 \\
3 & 3.77 & 315 \\
4 & 2.78 & 312 \\
5 & 2.68 & 299 \\
6 & 2.03 & 254 \\
7 & 1.99 & 1.9
\end{tabular}

Einstellung von unten; Konstanz 2-15 Stunden.

b) Isotherme zwischen $\mathrm{MnJ}_{2} \cdot 2 \mathrm{NH}_{3}$ und $\mathrm{MnJ}_{2}$.

Heizflüssigkeit Naphthalin rom Siedepunkt $214.8^{\circ}(707 \mathrm{~mm})$.

Tabelle 12.

$\mathrm{MnJ}_{2} \cdot 2 \mathrm{NH}_{3} \rightleftharpoons \mathrm{MnJ}_{2}+2 \mathrm{NH}_{2}$.

\begin{tabular}{c|c|c}
\hline \hline Nr. & Mole $\mathrm{NH}_{3}$ & $p$ mm korr. \\
\hline \hline 1 & 1.99 & 16.5 \\
2 & 1.80 & 14.6 \\
3 & 1.43 & 14.6 \\
4 & 1.00 & 14.6
\end{tabular}


Eine zweite derartige Versuchsreihe wurde mit besonderer Sorgfalt durchgeführt: Das Ausgan gsmaterial, Hexammin, war zunächst 10 Tage im Hochvakuumexsikkator über Ätzkali und Salmiak getrocknet worden. Um die während des Einwägens angezogene Feuchtigkeit zu beseitigen, wurde im Tensionsapparat selbst nochmals 24 Stunden im Vakuum getrocknet. Dann erst begann der Abbau zunächst sehr vorsichtig bei $50^{\circ}$, dann in Toluoldampf und dann erst die eigentliche Versuchsreihe mit Naphthalin als Heizflüssigkeit.

Tabelle 13.

\begin{tabular}{c|c||c|c|c}
\hline $\mathrm{Nr}$. & $\mathrm{g} \mathrm{MnJ}$ & $g \mathrm{NH}_{\mathrm{g}}$ & Mole $\mathrm{NH}_{3}$ & $p$ mm korr. \\
\hline \hline 1 & 0.5813 & 0.0541 & 1.69 & 14.4 \\
2 & $n$ & 00429 & 1.34 & 14.4 \\
3 & $"$ & 0.0194 & 0.606 & 14.4
\end{tabular}

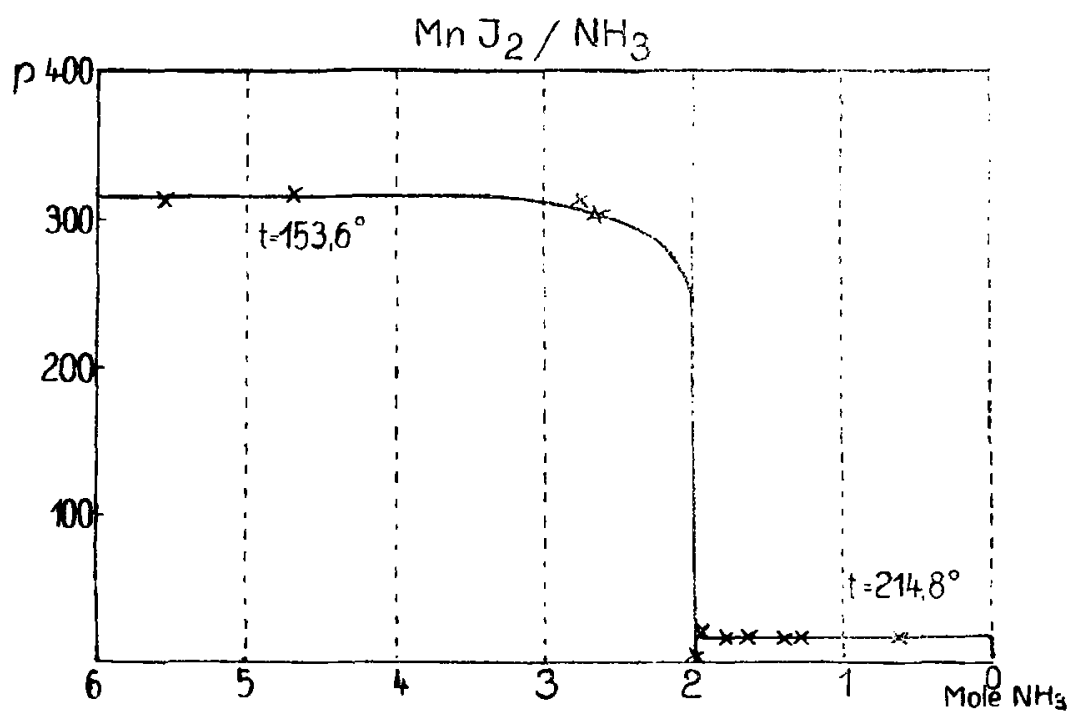

Fig. 3.

So wurde erreicht, daB die Probe auch während des Abbaues zunächst rein weiß blieb, während das Präparat von Tabelle 11 sich bräunlich-gelb gefärbt hatte. Das Diammin erschien in mikroskopischen Skeletten der ursprünglichen Oktaeder von rein weiBer Farbe. Erst als die Konzentration des Diammins unterschritten war, trat 
als Bodenkörper das Manganjodür auf und zwar rein rosa ${ }^{1}$ ) aber noch tiefer als das Bromid gefärbt. Der nach völligem Abpumpen des Ammoniaks im Tensionsapparat verbleibende Rückstand hatte gegenüber der Einwage $0.1930 \mathrm{gr}$ an Gewicht verloren während sich für den vollkommenen Abbau von Hexammin $0.1924 \mathrm{~g}$ berechnen. Er bestand demnach aus reinem Manganjodür.

Einen Überblick über die Existenzgebiete des Hexamminmanganojodids und Diammin manganojodids gibt Fig. 3, die zugleich zeigt, daB ein Monammin hier nicht besteht. Das gleiche müBte man schon aus dem Auftreten der rosa Färbung des Manganjodürs beim Beginn des Diammin abbaues schlieBen.

c) Tensionswerte von $\mathrm{MnJ}_{2} \cdot 6 \mathrm{NH}_{3}$.

Tabelle 14.

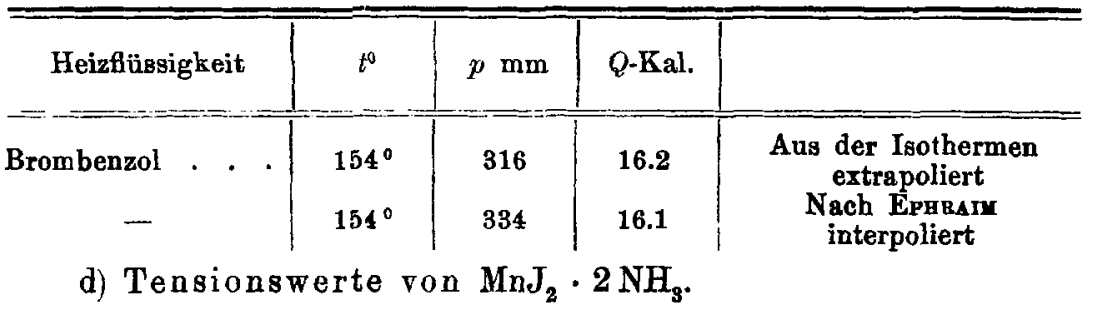

Tabelle 15.

\begin{tabular}{|c|c|c|c|c|}
\hline Heizflüssigkeit & $t^{0}$ & $p \mathrm{~mm}$ & $Q$-Kal. & \\
\hline $\begin{array}{l}\text { Nitrobenzol } \\
\text { Naphthalin }\end{array}$ & $\begin{array}{l}208^{\circ} \\
215^{\circ}\end{array}$ & $\begin{array}{l}11.1 \\
14.6\end{array}$ & $\begin{array}{l}21.6 \\
21.7\end{array}$ & Aus der Isothermen \\
\hline
\end{tabular}

\section{Eisenchlorür-Ammoniakate.}

Wasserfreies Eisenchlorür wurde entweder nach der beim Manganchlorür beschriebenen Art aus einem KaHLBAUMschen wasserhaltigen Präparat als hellgraugelbliches Pulver gewonnen:

$$
\begin{array}{lll}
\text { 1. } & 0.4490 \mathrm{~g} \text { gaben } 1.0125 \mathrm{~g} \mathrm{AgCl} ; 55.8 \% & \mathrm{Cl} \\
\text { 2. } & 0.1683 \mathrm{~g} \text { gaben } 0.3783 \mathrm{~g} \mathrm{AgCl} ; 55.6 \% & \mathrm{Cl} \\
\text { ber. } 55.9 \% & \mathrm{Cl}
\end{array}
$$

Oder, als reinweißes Präparat, nach H. u. W. Brutz").

1) $\mathrm{DaB}_{\mathrm{a}}$ die Farbe der wasserfreien Salze, wie hier in der Manganreihe, mit der der Lösungen übereinstimmt, ist eine seltene, sonst eigentlich nur beim Praseodym nnd Neodym geläufige Tatsache.

2) Übungsbeispiele aus der unorganischen Experimentalchemie, Leipzig bei W. Enamuman, II. Aufl, 1913, S. 67. 
Da Girardet das Gebiet zwischen Hexammin und Diammin bereits abgesucht hatte, blieb nur übrig, auf ein Monammin zu prüfen. Hierzu wurde ein Hexammin entsprechend abgebaut und vom Diammin aus der Druck gemessen.

a) Isotherme $\mathrm{z}$ wischen $\mathrm{FeCl}_{2} \cdot 2 \mathrm{NH}_{3}, \mathrm{FeCl}_{2} \cdot \mathrm{NH}_{3}$ und $\mathrm{FeCl}_{2}$, Heizflüssigkeit $\alpha$-Brom a a ph thalin vom Siedepunkte $276.7^{\circ}(700 \mathrm{~mm})$.

Tabelle 16.

$\mathrm{FeCl}_{2} \cdot 2 \mathrm{NH}_{8} \rightleftharpoons \mathrm{FeCl}_{\mathrm{g}} \cdot \mathrm{NH}_{3}+\mathrm{NH}_{\mathrm{s}} \rightleftharpoons \mathrm{FeCl}_{8}+2 \mathrm{NH}_{8}$.

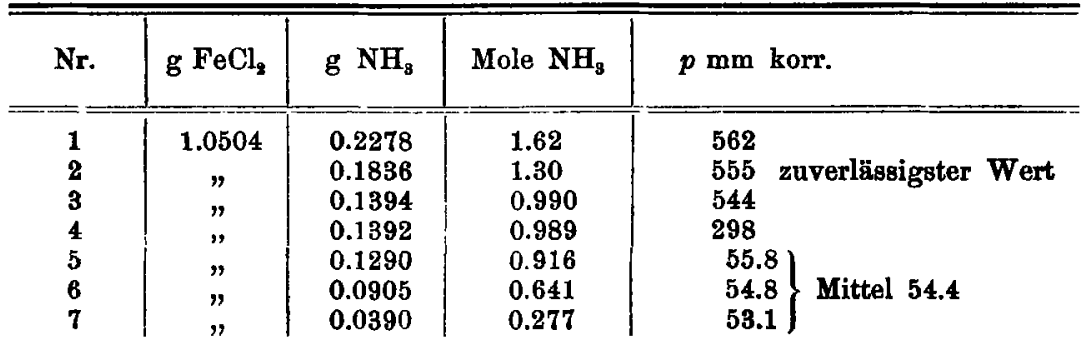

Einstellung stets von unten; sie erfolgte bei den letzten Werten in wenigen Minuten, bei den ersten etwas langsamer. Konstanz $1 / 2$ bis 14 Stunden. Die Farbe der Präparate ist grau. Die Existenz des Monoamminferro. chlorids ergibt sich aus Fig. 4 mit voller Schärfe.

b) Tensionswerte von $\mathrm{FeCl}_{2} \cdot 6 \mathrm{NH}_{3}$.

Wir geben im nachstehenden einige Vergleichswerte von GIRARDET und EpHrarm und die nach der Näherungsgleichung berechneten Zahlen für $Q$. Die beiderseitigen $p / t$-Kurven schneiden sich bei etwa $85^{\circ}$.

Tabelle 17.

\begin{tabular}{|c|c|c|c|c|}
\hline Nr. & $t^{0}$ & $p \mathrm{~mm}$ & \multicolumn{2}{|c|}{$Q$-Kal. } \\
\hline $\begin{array}{l}1 \\
2 \\
3 \\
4 \\
5\end{array}$ & $\begin{array}{c}90^{\circ} \\
106^{\circ} \\
93^{\circ} \\
105.7^{\circ} \\
115^{\circ}\end{array}$ & $\begin{array}{l}\mathbf{2 7 0} \\
\mathbf{5 6 3} \\
\mathbf{2 7 5} \\
\mathbf{4 9 0} \\
\mathbf{7 1 5}\end{array}$ & $\left.\begin{array}{l}13.65 \\
13.8 \\
13.8 \\
13.85 \\
13.9\end{array}\right\}$ & $\begin{array}{l}\text { Beobachter } \\
\text { GIRARDIT } \\
\text { Beobachter } \\
\text { EPHAMI }\end{array}$ \\
\hline
\end{tabular}

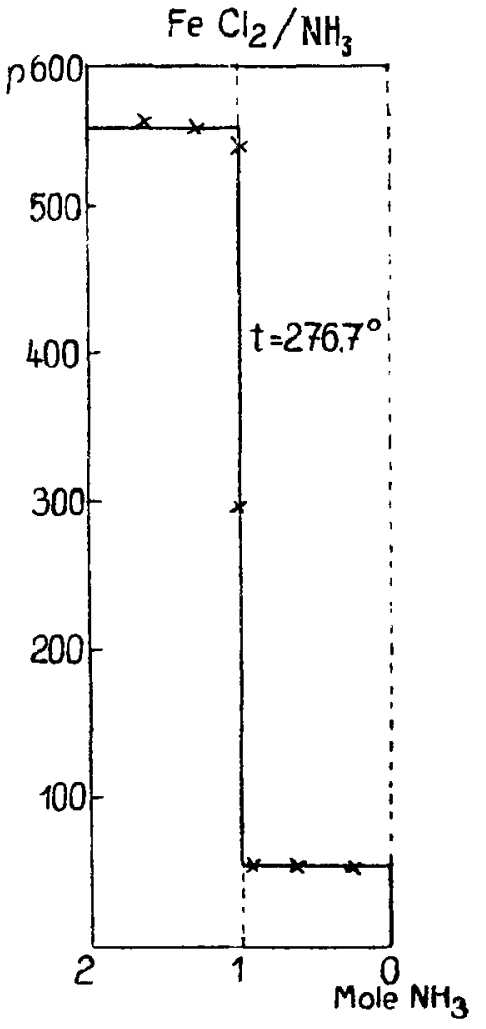

Fig. 4. 
c) Tensionswerte von $\mathrm{FeCl}_{2} \cdot 2 \mathrm{NH}_{3}$.

Tabelle 18.

\begin{tabular}{l|c|c|c}
\hline \hline Heizfüssigkeit & $t^{0}$ & $p \mathrm{~mm}$ & $Q$-Kal. \\
\hline 1. Thymol . . . . . & $230^{\circ}$ & $121(180)$ & 20.3 \\
2. $\alpha$-Bromnaputhalin . . & $277^{\circ}$ & $555(>800)$ & 20.7
\end{tabular}

Die erste Angabe stellt einen Mittelwert aus zwei innerhalb eines Millimeters stimmenden unabhängigen Versuchen dar, von denen der eine von oben, der andere von unten eingestellt wurde; die Drucke waren bis 14 Stunden konstant geblieben. Der zweite ist der zuverlässigste Wert der Isothermen. Die Konzentration lag bei 1.2 bis $1.3 \mathrm{Mol} \mathrm{NH}_{3}$. Die in der Tabelle geklammerten Werte von Girardet sind danach zweifellos zu hoch.

d) Tensionswerte von $\mathrm{FeCl}_{2} \cdot \mathrm{NH}_{3}$.

Tabelle 19.

\begin{tabular}{l|c|r|l}
\hline \hline Heizflüssigkeit & $t^{\bullet}$ & $p \mathrm{~mm}$ & $Q-\mathrm{Kal}$. \\
& & & \\
\hline \hline 1. Naphthalin . . . & $214.5^{\circ}$ & 5.8 & 22.5 \\
2. Thymol & $230^{0}$ & 10.9 & 22.7 \\
3. $\alpha$.Bromnuphthalin . & $277^{\circ}$ & 54.4 & 23.1 Mittelwert aus der Isothermen
\end{tabular}

Wie einleitend bemerkt, verbinden sich nach EpHrarm mit Eisenchlorür bei Zimmertemperatur von Methylamin nur 5 Mole. Wir lieBen im Tensionsapparat aut reines Eisenchlorür in einer Kältemischung von fester Kohlensäure und Alkohol flüssiges Methylamin 6 Stunden einwirken. Man lieB dann während 2 Stunden das Gemisch in einer Eis-Kochsalzmischung und entfernte schlieblich bei Zimmertemperatur das überschüssige Metbylamin durch Abpumpen, doch so, daB der Druck niemals unter $700 \mathrm{~mm}$ sank. Der Druck änderte sich schlieBlich während 18 Stunden nicht mehr, als den Schwankungen der Zimmertemperatur entsprach, so daB also das Präparat als mit Methylamin von Atmosphärendruck bei Zimmertemperatur gesättigt gelten mub.

$0.5574 \mathrm{~g} \mathrm{FeCl}_{2}$ addierten $0,8160 \mathrm{~g} \mathrm{NH}_{2} \cdot \mathrm{CH}_{3} ; 59.4 \% ; 5.98 \mathrm{Mole}^{\mathrm{NH}_{2}} \cdot \mathrm{CH}_{3}$ ber. für $\mathrm{FeCl}_{2} \cdot 6 \mathrm{NH}_{z} \cdot \mathrm{CH}_{3}: 59.5 \%$

Über den Abbau dieses Hexamethylaminferrochlorids orientiert der nachfolgende Versuch.

e) Is o therme von $\mathrm{FeCl}_{2} \cdot 6 \mathrm{NH}_{2} \cdot \mathrm{CH}_{3}$. Heizflüssigkeit Benzol rom Siedepunkte $78.1(712 \mathrm{~mm})$. 
Tabelle 20.

\begin{tabular}{|c|c|c|c|c|}
\hline Nr. & g FeCl & g $\mathrm{NH}_{2} \cdot \mathrm{CH}_{3}$ & Mole $\mathrm{NH}_{2} \cdot \mathrm{CH}_{3}$ & $p$ mm korr. \\
\hline $\begin{array}{l}1 \\
2\end{array}$ & $\begin{array}{c}0.5574 \\
, y\end{array}$ & $\begin{array}{l}\left.0.7378^{1}\right) \\
0.5253\end{array}$ & $\begin{array}{l}5.41 \\
3.85\end{array}$ & $\begin{array}{l}310.5 \\
282\end{array}$ \\
\hline
\end{tabular}

Der Druck sinkt also, wie bei den Ammoniakrerbindungen längs der Isothermen etwas, was, wie dort, durch das Auftreten von Mischkristallen erklärt wird; bei 2.4 Mol liegt $p$ noch über $200 \mathrm{~mm}$, bei etwa $1.94 \mathrm{Mol}$ sinkt der Druck auf etwa $6 \mathrm{~mm}$. Danach liegen die Verhältnisse genau so, wie bei den Ammoniakaten, and es existiert als nächste Abbaustufe eine Dimethylaminverbindung des Eisenchlorürs. Extrapoliert man aus den Werten unserer Isothermen auf die Konzentration $6 \mathrm{Mol}$, so findet man $p=320 \mathrm{~mm}$. Aus den EpHratmschen Messungen folgt für die gleiche Temperatur $p=384 \mathrm{~mm}$. Aus dem Wertepaar: $t=78^{\circ}, p=320 \mathrm{~mm}$ und der chemischen Konstanten für Methylamin $3.35^{2}$ ) folgt nach der Näherungsgleichung $Q=13.1 \mathrm{Kal}$. Um die wirkliche Dissoziationswärme zu finden, müBte man diesen Wert nach der in der nachfolgenden Abhandlung beschriebenen Art korrigieren. Die Hexamethylaminverbindung ist voluminös und rein weiB; niedere Abbauprodukte erschienen grau bis schwarz.

\section{Б. Eigenbromür-Ammoniakate.}

Wasserfreies Eisenbromür wurde durch Trocknen des KAHLBAJMschen Präparates im Hochvakuum über Ätznatron bei Zimmertemperatur erhalten.

$$
\begin{array}{r}
0.2950 \mathrm{~g} \text { gaben } 0.5114 \mathrm{~g} \mathrm{AgBr} ; 73.8 \% \mathrm{Br} \\
\text { ber. } 74.1 \% \mathrm{Br}
\end{array}
$$

Die Sättigung des im Tensionsapparate nochmals nachgetrockneten Präparats mit Ammoniak erfolgte bei Zimmertemperatur ziemlich schnell.

$0.5704 \mathrm{~g} \mathrm{FeBr}_{2}$ addierten $0.2627 \mathrm{~g} \mathrm{NH}_{3} ; 31.5 \% ; 5.84 \mathrm{Mol} \mathrm{\textrm {NH } _ { 8 }}$ ber. für $\mathrm{FeBr}_{2} \cdot 6 \mathrm{NH}_{3}: 32.1 \%$

1) Für die Bestimmung der Zusammensetzung des Bodenkörpers wurde hier nicht gewogen, sondern nach einem von G. F. Hürria ausgearbeiteten Verfahren volumetrisch gemessen. Näheres darüber wird später veröffentlicht werden.

2) Berechnet nach Nernst, Theoret. und experimet. Grundlagen des neuen Warmesatzes. Halle a. S. bei W. Knapp, 1918, S. 111, Gleichung (89) und (90). 
a) Isotherme zwischen $\mathrm{FeBr}_{2} \cdot 6 \mathrm{NH}_{3}$ und $\mathrm{FeBr}_{2} \cdot 2 \mathrm{NH}_{3}$. Heizflüssigkeit Toluol vom Siedepunkt $107.0^{\circ}$ (700 mm).

Tabelle 21.

$\mathrm{FeBr}_{2} \cdot 6 \mathrm{NH}_{8} \rightleftharpoons \mathrm{FeBr}_{9} \cdot 2 \mathrm{NH}_{8}+4 \mathrm{NH}_{3}$.

\begin{tabular}{c|c|c|c|c}
\hline $\mathrm{Nr}$. & $\mathrm{g} \mathrm{FeBr}$ & $\mathrm{g} \mathrm{NH}$ & Mole NH, & $p$ mm korr. \\
\hline 1 & 0.5704 & nahezu 0.2627 & nahezu 6 & 126 \\
2 & $"$ & 0.2432 & 5.41 & 124 \\
3 & $"$ & 0.1018 & 2.26 & 122 \\
4 & $"$ & 0.0876 & 1.95 & 93.5 \\
5 & $"$ & 0.0854 & 1.90 & 3.3
\end{tabular}

Bei Nr. 1-4 betrug die Druckkonstanz jeweils mehrere Stunden.

b) Isotherme $z$ wischen $\mathrm{FeBr}_{2} \cdot 2 \mathrm{NH}_{3}, \mathrm{FeBr}_{2} \cdot \mathrm{NH}_{3}$ und $\mathrm{FeBr}$. Heizflüssigkeit Thymol vom Siedepunkt $\mathbf{2 3 0 . 0}^{\circ}$ (709 mm).

Tabelle 22.

$\mathrm{FeBr}_{9} \cdot 2 \mathrm{NH}_{3} \rightleftharpoons \mathrm{FeBr}_{2} \cdot \mathrm{NH}_{3}+\mathrm{NH}_{3} \rightleftharpoons \mathrm{FeBr}_{2}+2 \mathrm{NH}_{3}$.

\begin{tabular}{c|c|c|c|c}
\hline Nr. & g FeBr & $\mathrm{g} \mathrm{NH}_{8}$ & Mole NH, & $p$ mm korr. \\
\hline 1 & 0.8012 & 0.1057 & 1.67 & 23 \\
2 & 0.2600 & 0.0297 & 1.45 & 24 \\
3 & 0.8012 & 0.0581 & 0.92 & 21 \\
4 & 0.5704 & 0.0370 & 0.82 & 9.4 \\
5 & 0.8012 & 0.0028 & 0.04 & 10
\end{tabular}

Druck 1 und 5 wurden von oben, die übrigen von unten eingestellt, Druckkonstanz meist über 10 Stunden. Das Hexamminferrobromid erscheint rein weiB, das Diamminferrobromid schmutziggrau, das Monoamminfer r obromid dunkelgrau. Das ammoniakfreie Bromür war leuchtend okergelb. Die Übersicht über die Existenzgebiete gibt Figur 5.

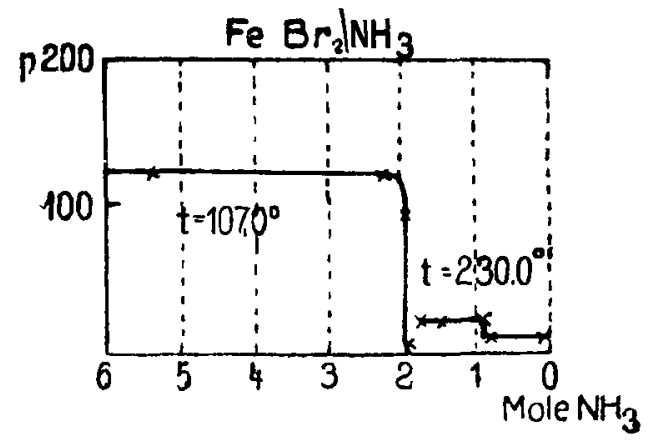

Fig. 5. 
c) Tensionswerte von $\mathrm{FeBr}_{2} \cdot 6 \mathrm{NH}_{3}$.

Tabelle 23.

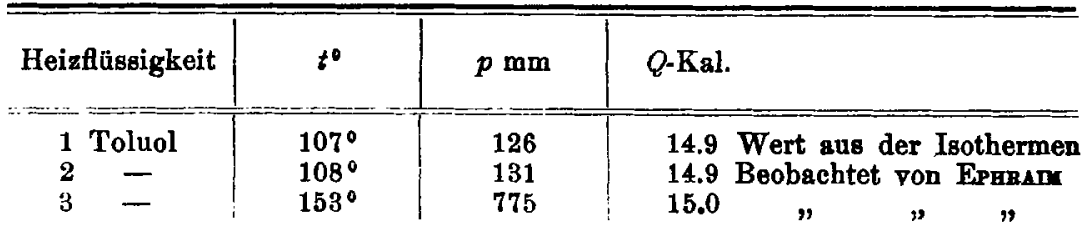

d) Tensionswerte von $\mathrm{FeBr}_{2} \cdot 2 \mathrm{NH}_{3}$.

Tabelle 24.

\begin{tabular}{|c|c|c|c|}
\hline Heizflüssigkeit & $t^{0}$ & $p \mathrm{~mm}$ & $Q$-Kal. \\
\hline $\begin{array}{l}\text { Naphthalin . } \\
\text { Thymol : } \\
\alpha \text {-Bromnaphthalin }\end{array}$ & $\begin{array}{l}\mathbf{2 1 5 ^ { \circ }} \\
\mathbf{2 3 0 ^ { \circ }} \\
\mathbf{2 7 7 ^ { \circ }}\end{array}$ & $\begin{array}{l}11.5 \\
23.5 \\
126\end{array}$ & $\begin{array}{l}21.9 \\
21.9 \text { Wert aus der Isothermen } \\
22.3\end{array}$ \\
\hline
\end{tabular}

Bei 1 und 3 enthielt der Bodenkörper 1.5 bis $1.7 \mathrm{Mol} \mathrm{NH}_{3}$. e) Tensionswerte von $\mathrm{FeBr}_{3} \cdot \mathrm{NH}_{3}$.

Tabelle 25.

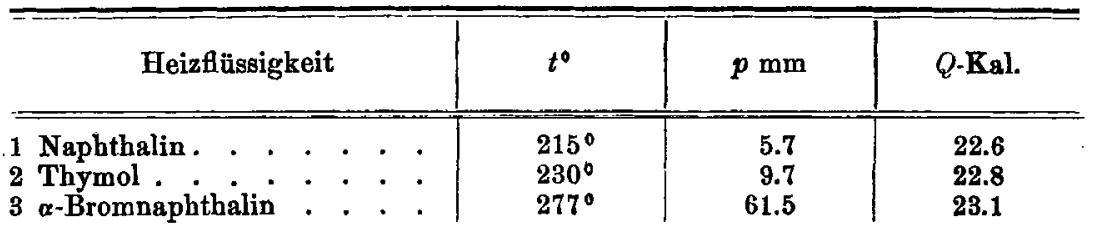

Bei 1 und 3 enthielt der Bodenkörper nur noch sehr wenig überschüssiges Ammoniak; Wert 2 entstammt der Isothermen und ist bei weitem der zurerlässigste.

\section{Eisenjodür-Ammoniakate.}

Versuche, Hexammin ferrojodür nach der beim Mangan befolgten Art auf nassem Wege zu bereiten, führten trotz Verbesserung des Luftabschlusses stets zu sauerstoffhaltigen Präparaten, obwohl zwar zunächst rein weiße, anscheinend zuverlässige Niederschläge entstanden. Wir benutzten als Ausgangsmaterial deshalb wasserfreies Eisenjodür, das wir nach $\mathrm{J}_{\text {ACKBON }}$ und DERBY ${ }^{1}$ ) darstellten, indem „ferrum reductum" innerbalb einer Eisenröhre in einem jodbeladenen Wasserstoffstrome erhitzt und das entstandene Ferrojodid als Sublimat in einer engeren, der ersten eingedichteten Eisenröhre auf-

1) C. L. JAcrson und J. H. Deriy, Amer. Chem. J. 24 (1899), 15. 
gefangen wurde. Stark spiegelnde, im auffallenden Lichte tiefschwarz, im durchfallenden Lichte granatrot erscheinende Blättchen, die sich im Wasser farblos lösten und nur eine Spur Trübung, vermutlich verstäubtes Eisen, hinterlieben.

$$
\begin{array}{r}
0.2082 \mathrm{~g} \text { gaben } 0.3140 \mathrm{~g} \mathrm{AgJ;} 81.5 \% \\
\text { ber. } 81.9 \%
\end{array}
$$

Die Einwage zur Herstellung des Hexammins wurde gemäB dieser Analyse auf den Gehalt der Probe an $\mathrm{FeJ}_{2}$ korrigiert. Es verschluckten im Tensionsapparat bei Eiskühlung im Laufe von 24 Stunden:

$$
\begin{aligned}
& 0.8820 \mathrm{~g} \mathrm{FeJ} \\
& \text { ber. für } \mathrm{FeJ}_{2}, 6 \mathrm{gH}_{3}: 24.8 \% \text {. }
\end{aligned}
$$

Das Hexammin' ist höchst voluminös; das Präparat war weiß mit einem Stich ins gelbliche. Ganz reines Hexammin - vgl. den Versuch zur Darstellung auf nassem Wege - dürfte rein weiß sussehen.

a) Isotherme $\mathrm{z}$ wischen $\mathrm{FeJ}_{2} \cdot 6 \mathrm{NH}_{3}$ und $\mathrm{FeJ}_{2} \cdot 2 \mathrm{NH}_{3}$. Heizflüssigkeit Brombenzol vom Siedepunkt $1_{33.0^{\circ}}$ (702 mm).

\begin{tabular}{|c|c|c|c|c|}
\hline Nr. & $\mathrm{g} \mathrm{FeJ}_{2}$ & $\mathrm{~g} \mathrm{NH}$ & Mole $\mathrm{NH}_{\mathrm{B}}$ & $p$ mm korr. \\
\hline $\begin{array}{l}1 \\
2 \\
3 \\
4 \\
5\end{array}$ & $\begin{array}{c}0.8820 \\
" \\
" \\
"\end{array}$ & $\begin{array}{l}0.2610 \\
0.1640 \\
0.1062 \\
0.0955 \\
0.0942\end{array}$ & $\begin{array}{l}5.39 \\
3.39 \\
2.19 \\
1.97 \\
1.94\end{array}$ & $\begin{array}{r}205 \\
201 \\
197 \\
103 \\
2.6\end{array}$ \\
\hline
\end{tabular}

Tabelle 26.

$$
\mathrm{FeJ}_{2} \cdot 6 \mathrm{NH}_{2} \rightleftharpoons \mathrm{FeJ}_{2} \cdot 2 \mathrm{NH}_{3}+4 \mathrm{NH}_{2} \text {. }
$$

Die Einstellung erfolgte von unten. Die Farbe des Hexammins ändert sich während des Abbaues in rosa bis schmutzig ziegelrot. Das Diamminferrojodid tritt als sepiabraune Phase auf; dabei schwindet der Bodenkörper sehr stark.

b) Isotherme zwischen $\mathrm{FeJ}_{2} \cdot 2 \mathrm{NH}_{3}$ und $\mathrm{FeJ}_{2}$. Heizflüssigkeit Naphthalin rom Siedepunkt $214.7^{\circ}(705 \mathrm{~mm})$.

Tabelle 27.

$$
\mathrm{FeJ}_{2} \cdot 2 \mathrm{NH}_{3} \rightleftharpoons \mathrm{FeJ}_{2}+2 \mathrm{NH}_{2} \text {. }
$$

\begin{tabular}{c|c|c|c|c}
\hline Nr. & g FeJ, & $\mathrm{g} \mathrm{NH}_{3}$ & Mole $\mathrm{NH}_{3}$ & $p$ mm korr. \\
\hline 1 & 0.5194 & 0.0489 & 1.72 & 6.1 \\
2 & $"$ & 0.0310 & 1.09 & 6.0 \\
3 & $"$ & 0.0085 & 0.30 & 6.0
\end{tabular}


Der Druck unter 1 und 2 wurde von oben, Druck 3 von unten erreicht.

Ein Monoamminferrojodid existiert unter unseren Versuchsbedingungen im Gleichgewichte mit Ammoniakgas ebensowenig, wie eine solche Verbindung des Mangans, Kobalts und Nickels. Vgl. Figur 6.

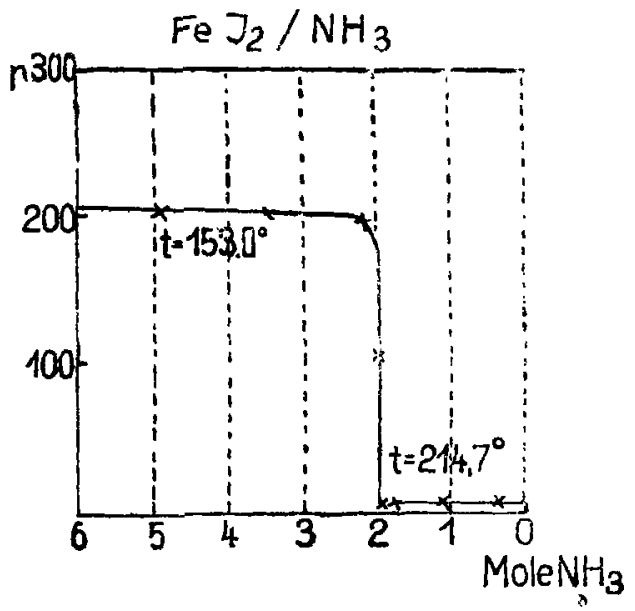

Fig. 6.

c) Tensionswerte ron $\mathrm{FeJ}_{2} \cdot 6 \mathrm{NH}_{3}$.

Tabelle 28.

\begin{tabular}{|c|c|c|c|}
\hline Heizflüssigkeit & $t^{0}$ & $p \mathrm{~mm}$ & $Q$-Kal. \\
\hline Brombenzol & $153^{\circ}$ & 206 & ert aus der Isothermen \\
\hline
\end{tabular}

d) Tensionswerte ron $\mathrm{FeJ}_{2} \cdot 2 \mathrm{NH}_{3}$.

Tabelle 29.

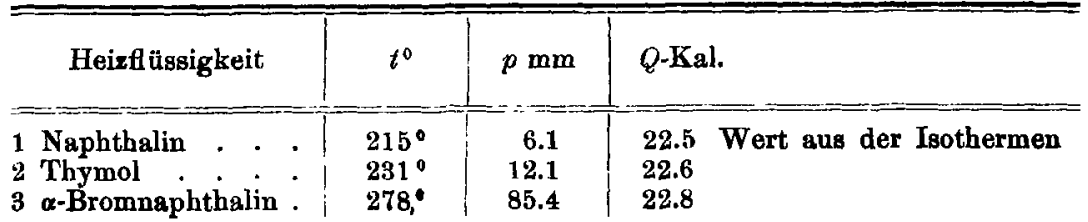

Von den untersuchten 16 Stoffen sind die 8 Verbindungen: $\mathrm{MnCl}_{2} \cdot \mathrm{NH}_{3}, \mathrm{MnBr}_{2} \cdot 2 \mathrm{NH}_{3}, \mathrm{MnBr}_{2} \cdot \mathrm{NH}_{3}, \mathrm{MnJ}_{2} \cdot 2 \mathrm{NH}_{3}, \mathrm{FeCl}_{2} \cdot \mathrm{NH}_{3}$, $\mathrm{FeBr}_{2} \cdot 2 \mathrm{NH}_{3}, \mathrm{FeBr}_{2} \cdot \mathrm{NH}_{3}, \mathrm{FeJ}_{2} \cdot 2 \mathrm{NH}_{3}$ hier zum ersten Male be- 
110 W. Biltz u. G. F. Hïttig. Ammonialverbindungen der Halogenide usw.

schrieben. Die auber diesen 16 Stoffen sonst noch in der Literatur erwähnten Verbindungen existieren in den abgesuchten Existenzgebieten im Gleichgewichte mit gasförmigem Ammoniak nicht und sind einstweilen zu streichen. Die Verhältnisse liegen denen bei Nickel und Kobalt völlig entsprechend. Eine Neuberechnung der Versuchszahlen der nun vollständigen Verbindungsreihe aus der Mangan-, Eisen-, Kobalt-, Nickel-Gruppe zum Zwecke besseren Vergleichs wird in der nachstehenden, der Vergleich selbst in der drittfolgenden Abhandlung vorgenommen werden.

Clausthal im Harw, Chemisches Laboratorium der Bergakademie.

Bei der Redaktion eingegangen am 5. August 1919. 